\title{
Recent Developments in Local Helioseismology
}

\author{
M.J. Thompson · S. Zharkov
}

Received: 14 November 2007 / Accepted: 7 February 2008 / Published online: 6 March 2008

(C) Springer Science+Business Media B.V. 2008

\begin{abstract}
Local helioseismology is providing new views of subphotospheric flows from supergranulation to global-scale meridional circulation and for studying structures and dynamics in the quiet Sun and active regions. In this short review we focus on recent developments, and in particular on a number of current issues, including the sensitivity of different measures of travel time and testing the forward modelling used in local helioseismology. We discuss observational and theoretical concerns regarding the adequacy of current analyses of waves in sunspots and active regions, and we report on recent progress in the use of numerical simulations to test local helioseismic methods.
\end{abstract}

Keywords Sun: local helioseismology $\cdot$ Sun: time-distance analysis $\cdot$ Sun: forward problem · Sun: inversion

\section{Introduction}

Helioseismology provides a remarkable capability to image beneath the surface of the Sun and thus to test our understanding of the physics of solar and stellar interiors. Through the study of properties of global modes, and in particular their frequencies, global-mode helioseismology has enabled us to image the radial variation of the Sun's hydrostatic structure through most of the interior as well as the radial and latitudinal variation of its rotation rate. However, the global-mode frequencies do not discriminate in the longitudinal direction, nor do they distinguish between northern and southern hemispheres. The global eigenfunctions can in principle be used to probe such variation, but the global modes are not the natural tool to explore such aspects. Rather, with the advent of high-spatial-resolution helioseismic observations of the solar surface, a number of different techniques have been developed, going

\section{Invited Review.}

Helioseismology, Asteroseismology, and MHD Connections

Guest Editors: Laurent Gizon and Paul Cally.

M.J. Thompson $(\bowtie) \cdot$ S. Zharkov

Department of Applied Mathematics, School of Mathematics and Statistics, University of Sheffield,

Hounsfield Road, Sheffield S3 7RH, UK

e-mail: michael.thompson@sheffield.ac.uk 
under the generic name of local helioseismology. Local helioseismic techniques are much better suited to the task of studying horizontally localised features than is global-mode helioseismology. These techniques are providing us today with subphotospheric maps of supergranulation (Duvall et al., 1997) and of flow patterns surrounding active regions (Gizon, Duvall, and Larsen, 2001; Haber et al., 2001; Zhao and Kosovichev, 2004). Local helioseismological methods help us detect large-scale azimuthal and meridional flows (Giles et al., 1997; Basu, Antia, and Tripathy, 1999) and to monitor active regions on the Sun's far surface (Lindsey and Braun, 2000). They have also allowed us to take a first look into internal structures beneath sunspots and active regions (e.g., Kosovichev, Duvall, and Scherrer, 2000).

The principal local helioseismic techniques in common use are time-distance helioseismology (Duvall et al., 1993), ring-diagram analysis (Hill, 1988), and helioseismic holography (Lindsey and Braun, 1997) and the closely related method of acoustic imaging (Chou, 2000). Each has a different conceptual basis. Ring-diagram analysis is closest to globalmode helioseismology: what is determined is the local dispersion relation of waves observed in a localised region of solar surface. Time-distance helioseismology works in terms of travel times of wave packets travelling between pairs of points on the solar surface; the wave packets are made manifest by cross-correlating the oscillatory signals at the surface points. Helioseismic holography reconstructs subsurface wave fields from surface observations, in particular the ingoing and outgoing waves with respect to a pupil, from which correlations can be measured and phase shifts derived. Other methods of local helioseismology, which shall not however be mentioned further in this paper, are Hankel spectral analysis (Braun, Duvall, and Labonte, 1987) and statistical waveform analysis (Woodard, 2002).

Local helioseismology has been excellently reviewed by Gizon and Birch (2005). Here we consider some of the developments in the field since then. We do not presume to a complete review of all such developments. Indeed we focus largely on time-distance helioseismology. For a recent short review of local helioseismology focussing on the techniques, see Birch (2006). A discussion of some outstanding issues in time - distance helioseismology is presented by Gizon and Thompson (2007).

\section{The Fundamental Observable of Time-Distance Helioseismology}

Time-distance helioseismology proceeds by cross-correlating wave-field measurements (typically Doppler velocities) at different locations on the solar surface. These crosscorrelations are then compared with a reference cross-correlation, obtained from a model.

The equations of solar oscillations may be combined in the form

$$
L[\xi]=\mathbf{s},
$$

where $L=\rho \partial_{t}^{2}+\cdots$ is a linear differential operator governing adiabatic oscillations and $\mathbf{s}$ is a stochastic source function. Operator $L$ depends on the solar interior parameters such as sound speed $(c)$, density $(\rho)$, bulk flow (u), magnetic field $(\mathbf{B})$, and damping. A seismic model is one that fits the observed data and is consistent with the estimated properties of the data errors. Given a set of observations, one task of helioseismology is to construct, if possible, a seismic model, that is, to obtain such a distribution of the underlying parameters that fits the totality of the observed data. The common approach to this task in local helioseismology is to make some basic assumptions about wave sources and then to consider the linearised perturbations to some initial model of the solar interior. The only perturbations commonly considered to date have been to introduce a flow and to allow a modification of the sound-speed distribution [i.e., the operator is perturbed from $L(c, \ldots ; \mathbf{u}=0)$ to $L(c+\delta c, \ldots ; \mathbf{u})]$. 


\subsection{Filtering}

By ignoring systematic errors such as instrumental and geometric artifacts (e.g., foreshortening), the line-of-sight wave velocity observed at some height $z=z_{\text {obs }}$ in the solar atmosphere can be written as

$$
\phi=\mathrm{PSF} *\left[\hat{\mathbf{n}} \cdot \partial_{t} \boldsymbol{\xi}\left(\mathbf{r}, z_{\mathrm{obs}}, t\right)\right],
$$

where $\mathbf{r}$ is a horizontal position vector on the solar surface defined by the height $z_{\mathrm{obs}}, t$ is time, $\hat{\mathbf{n}}$ is a unit vector pointing in the direction of the observer, and $\boldsymbol{\xi}$ is the wave displacement vector. Equation (2) also contains the convolution (indicated with the star operator) with the instrument point-spread function (PSF). In local helioseismology, a reference frame that is co-rotating with the Sun is normally used to remove the main component of the rotation.

Further, the oscillation signal may be filtered in the Fourier domain to obtain

$$
\psi(\mathbf{k}, \omega)=F(\mathbf{k}, \omega) \phi(\mathbf{k}, \omega),
$$

where $\mathbf{k}$ is the horizontal wave vector, $\omega$ is the angular frequency, and $F$ is a filter function chosen by the observer to remove granulation noise and to select parts of the wavepropagation diagram. These may include, but are not limited to, a filter selecting data on the $f$-mode ridge for working with $f$ modes, or removal of the $f$-mode ridge to leave acoustic modes only, combined with phase-speed filters (Duvall et al., 1997).

\subsection{Cross-Correlations and Travel Times}

The fundamental observable of time - distance analysis is the cross-correlation function between the filtered Doppler velocities at two surface locations $\mathbf{r}_{1}$ and $\mathbf{r}_{2}$ :

$$
C\left(\mathbf{r}_{1}, \mathbf{r}_{2}, t\right)=\frac{1}{T-|t|} \int \psi\left(\mathbf{r}_{1}, t^{\prime}\right) \psi\left(\mathbf{r}_{2}, t^{\prime}+t\right) \mathrm{d} t^{\prime},
$$

where $T$ is the duration of the observation. Positive time lags $(t>0)$ provide information about waves propagating from $\mathbf{r}_{1}$ to $\mathbf{r}_{2}$, whereas negative time lags $(t<0)$ give information about waves travelling in the opposite direction.

A seismic model in a strong sense would be a three-dimensional model of the solar interior that was consistent with the large set of observed cross-correlations. Full waveform modelling to fit the cross-correlations has not yet been attempted. Instead, only a few parameters are chosen to describe the behaviour of the cross-correlation. Indeed, commonly a single functional measure obtained from the cross-correlation is used: Travel time is the widely used parameter in time - distance helioseismology. We note in passing that the phase perturbations of helioseismic holography are also commonly expressed in terms of traveltime perturbations (see, e.g., Braun et al., 2007).

By expressing the wave field as a superposition of global normal-mode solutions for standing waves of a spherically symmetric Sun, Kosovichev and Duvall (1997) and Giles (1999) modelled the measured cross-correlation function as a Gabor wavelet, a fiveparameter analytic function incorporating the values of the central frequency $\left(\omega_{0}\right)$ of the wave packet, its width $(\Delta \omega)$, group travel time $\left(t_{\mathrm{g}}\right)$, and phase travel time $\left(t_{\mathrm{p}}\right)$, as well as its amplitude $(A)$ :

$$
G(\mathbf{p}, t)=A \exp \left[-\frac{\Delta \omega^{2}}{4}\left(t-t_{\mathrm{g}}\right)^{2}\right] \cos \left[\omega_{0}\left(t-t_{\mathrm{p}}\right)\right]
$$


By $\mathbf{p}$ we denote the vector of parameters $\left\{A, \Delta \omega, t_{\mathrm{g}}, \omega_{0}, t_{\mathrm{p}}\right\}$. Travel times are then obtained by fitting expression (5) to the measured cross-correlation function. Generally the phase travel-time parameter $\left(t_{\mathrm{p}}\right)$ is taken as the measured travel time and we shall do that here. A travel-time perturbation is then computed as the difference between the travel time so obtained and the corresponding travel time in an unperturbed medium, for example obtained from a Gabor-wavelet fit to the cross-correlation signal averaged over a region of quiet Sun. Nigam, Kosovichev, and Scherrer (2007) have recently provided a generalised formula for the Gabor wavelet, taking into account both the radial and horizontal components of the oscillation displacement signal as well as phase-speed filtering used in measuring the observed cross-correlation signal.

Motivated by studies in geophysics, Gizon and Birch $(2002,2004)$ have developed a definition of travel time that consists of linearising the difference between the observed cross-correlation signal and a sliding reference cross-correlation signal $\left(C^{\text {ref }}\right)$ :

$$
X_{ \pm}\left(\mathbf{r}_{1}, \mathbf{r}_{2}, \tau\right)=\int_{-\infty}^{\infty} \mathrm{d} t^{\prime} f\left( \pm t^{\prime}\right)\left(C\left(\mathbf{r}_{1}, \mathbf{r}_{2}, t^{\prime}\right)-C^{\mathrm{ref}}\left(\mathbf{r}_{1}, \mathbf{r}_{2}, t^{\prime} \mp \tau\right)\right)^{2}
$$

Here $f( \pm t)$ is a window function designed to separate waves travelling in different directions (i.e., positive and negative branches of the cross-correlation function) and also different bounces for the $p$ modes. The travel-time perturbation for waves travelling between $\mathbf{r}_{1}$ and $\mathbf{r}_{2}$ is then defined as the value of $\tau$ that minimises $X_{ \pm}$, which leads to the following formula for computing travel-time perturbations:

$$
\tau_{ \pm}=\frac{\sum_{i} \mp f\left( \pm t_{i}\right) \dot{C}^{\mathrm{ref}}\left(t_{i}\right)\left[C\left(t_{i}\right)-C^{\mathrm{ref}}\left(t_{i}\right)\right]}{\sum_{i} f\left( \pm t_{i}\right)\left[\dot{C}^{\mathrm{ref}}\left(t_{i}\right)\right]^{2}}
$$

where the dots denote derivatives with respect to time. The travel-time perturbation can be expressed as a summation over the values at the discrete (presumed uniformly spaced) times $\left(t_{i}\right)$ at which the observations were made.

The reference cross-correlation function $\left(C^{\text {ref }}\right)$ may be calculated by solving the hydrodynamic equations in a solar model with stochastically distributed sources (Gizon and Birch, 2002; Birch, Kosovichev, and Duvall, 2004). Indeed, one attractive feature of the GizonBirch definition of travel time is that it allows an immediate interpretation in terms of perturbations to a reference solar model. Alternatively, as for some of the results presented later in Section 4, the reference cross-correlation can be obtained from observation as an average cross-correlation over a region of quiet Sun.

To gain some insight into what the Gizon - Birch method's travel-time measure is actually sensitive to, one may consider applying it in the case where a simulated cross-correlation function and the reference cross-correlation both take the form of Gabor wavelets. Then, neglecting the effect of the window function, and using Equation (5) and the continuous version of Equation (7), one can show that

$$
\tau_{+} \approx-\int_{-\infty}^{\infty} \frac{\partial G}{\partial \mathbf{p}} \cdot \delta \mathbf{p} \frac{\partial G}{\partial t} \mathrm{~d} t / \int_{-\infty}^{\infty}\left(\frac{\partial G}{\partial t}\right)^{2} \mathrm{~d} t
$$

where $G$ and $\mathbf{p}$ are the same as in Equation (5). By considering the response of $\tau_{+}$to individual perturbations of the parameters contained in $\mathbf{p}$, it can be shown that to first order the perturbations to amplitude $(A)$ and to wave packet width $(\Delta \omega)$ have no effect on the travel-time perturbation obtained via the Gizon - Birch definition, whereas the response to a 
perturbation in phase or group travel times would be

$$
\tau_{+}=\delta t_{\mathrm{p}}+\left(\delta t_{\mathrm{p}}-\delta t_{\mathrm{g}}\right) \frac{\Delta \omega^{2}}{\Delta \omega^{2}+4 \omega_{0}^{2}} .
$$

Note that in practice the second term is small ( $\Delta \omega$ generally being rather smaller than $\left.\omega_{0}\right)$, so that the measured travel-time perturbation is approximately equal to the perturbation in the phase travel time. Finally, a perturbation $\left(\delta \omega_{0}\right)$ to the central frequency $\left(\omega_{0}\right)$ of the Gabor wave packet would also introduce changes to the travel-time measured by the Gizon - Birch method if the waves were dispersive:

$$
\tau_{+} \approx\left(t_{\mathrm{p}}-t_{\mathrm{g}}\right) \frac{4 \omega_{0}^{2}}{\Delta \omega^{2}+4 \omega_{0}^{2}} \frac{\delta \omega_{0}}{\omega_{0}} .
$$

An observational comparison between the results of applying the two travel-time definitions - Gabor-wavelet fitting and Gizon - Birch - has recently been made (Roth, Gizon, and Beck, 2007). Using a SOHO/MDI Structure Program 72-hour times series of Dopplergrams for the quiet Sun, the study shows good agreement between the inferred travel times for the two methods of measurement.

\section{Modelling}

Forward modelling is the process of computing the wave field or some of its observable properties, based on a prescribed model of the solar structure and, for example, the background flow, magnetic field, or mode physics (excitation, damping, etc.). We shall refer to all of these as background properties. In general, changes to the background properties will result in changes to the wave field and its observable properties. An outcome of the modelling is often a linear-response kernel, which quantifies how a small change in background properties will change some observable property. The change in observable property is then expressible as a convolution of the kernel and the changes in background properties. The formulation of helioseismic linear-response kernels using the Born approximation was first presented by Birch and Kosovichev (2000) and using the Rytov approximation by Jensen and Pijpers (2003).

Gizon and Birch had the insight to relate the changes in background properties to changes in the fundamental observable of time - distance helioseismology, namely the crosscorrelation function. From there, one can compute as required the kernel relating those changes to the change in, say, the measured travel time. Following such a procedure, the linearised relationship between changes in the background properties and changes in, for example, the travel time $(\tau)$ can be written as

$$
\tau=\sum_{\alpha} \int K_{\alpha} \delta q_{\alpha} \mathrm{d} V
$$

where $\alpha$ labels different background properties $q_{\alpha}$ whose change may affect the observable. Thus the contribution that each perturbation $\left(\delta_{q}\right)$ makes to the travel-time perturbation $(\tau)$ is a convolution of that $\delta q_{\alpha}$ with a kernel $K_{\alpha}$. 


\subsection{Empirical Test of Kernel Calculations}

The linear-response kernels have been computed from theoretical considerations. Recently, however, Duvall, Birch, and Gizon (2006) have devised a means of estimating empirically the linear-response kernel in a particular circumstance. They have measured the mean traveltime perturbations for $f$ modes in the vicinity of isolated, small magnetic features. A small magnetic feature may perturb the travel times through various physical effects, but we assume that all of the contributions can be parameterised by a single parameter,

$$
\delta q_{\alpha}=c_{\alpha} \delta q
$$

where the vector of coefficients $c_{\alpha}$ is the same for all magnetic elements. Hence

$$
\tau=\int K \delta q \mathrm{~d} V
$$

where $K=\sum_{\alpha} c_{\alpha} K_{\alpha}$. This convolution may be written conveniently in Fourier space, where it simply becomes a product:

$$
\tau(\mathbf{k})=K(\mathbf{k}) \delta q(\mathbf{k}) .
$$

We have used the same symbols for quantities and their Fourier transforms: The latter are distinguished by being written explicitly as functions of the horizontal wavenumber $(\mathbf{k})$.

In the analysis of Duvall, Birch, and Gizon (2006) the single perturbative parameter $(\delta q)$ is the absolute photospheric magnetic field strength. Since the travel-time perturbations can be measured in the vicinity of many such isolated magnetic features, the Fourier-transformed kernel can then be estimated by making a fit of expression (14) to the observed magnetograms $(\delta q)$ and travel-time perturbations $(\tau)$, for each $\mathbf{k}$ separately.

Of course the resulting kernel corresponds to whatever combination of physical effects is present in the ensemble of magnetic features. The resulting kernels obtained by Duvall, Birch, and Gizon show close similarity to the theoretical kernels for wave damping computed by Gizon and Birch (2002).

The results from this new technique are fascinating as they are the first empirical verification of the kernels produced from theory. The $f$-mode kernels are essentially two dimensional, because the $f$ modes are confined close to the surface. By contrast, $p$-mode kernels are intrinsically three dimensional. The technique devised by Duvall, Birch, and Gizon (2006) can be extended to $p$ modes. However, because the perturber is confined to the near-surface layers (the ratio of magnetic to gas pressure presumably falling off very quickly with increasing depth), the results will only provide the kernels evaluated at the surface.

\subsection{Validity of Time-Distance Kernels for Flows}

Jackiewicz et al. (2007) have made a careful study of flow-speed kernels for interpreting $f$-mode travel-time data. One question that they address is the range of flow speeds in which the linear approximation is valid. By considering a uniform flow velocity (u), and making an expansion in powers of the small parameter $(\mathbf{u} \cdot \mathbf{k})$, they find that the linearisation of travel times with the flow amplitude is valid to within $10 \%$ for flow amplitude less than $250 \mathrm{~m} \mathrm{~s}^{-1}$ and travel distances less than $25 \mathrm{Mm}$. For larger distances or flow speeds, nonlinear effects become important. The same authors further deduce that the main effect on travel times of the flow is its advection of the waves: Advection of the sources may be neglected to a very good approximation when calculating the kernels. 
Jackiewicz et al. also show that, at small spatial scales, the kernels for Doppler velocity measurements depend significantly on position on the solar disk and on the angle between the observation points and the center-to-limb direction. This means that the kernels are not translationally invariant and so the inverse problem is not simply a deconvolution. Close to disk centre one may hope that translational invariance is still a reasonable approximation.

\subsection{Ring-Analysis Kernels}

It is assumed in inversions of ring-analysis data that the measured ring parameters are sensitive only to conditions directly beneath the region in which the observations were made. Hindman et al. (2005) have sought to test this assumption by a series of forward-modelling experiments. The regions of observation are typically rectangular "tiles," and within each tile a circular apodization is normally applied to the data before the power spectrum is calculated. Hindman et al. indeed find that the sensitivity of the ring parameters to various flows is essentially zero outside the tile and is proportional to the apodization function within the tile. These results have been confirmed by the recent work of Birch et al. (2007), who have computed the linear-response kernels of ring parameters to flows. Specifically, they calculate the linear sensitivity to small changes in the local power spectrum and then compute the sensitivity of the power spectrum to time-independent weak local flows. By combining these two results, they obtain the three-dimensional kernels that give the linear sensitivity of ring parameters to both vertical and horizontal flows. The sensitivity to flows is essentially limited to the region where the apodization is nonzero, and the depth dependence of the kernels is very close to the mode's kinetic-energy density.

\section{Helioseismology of Sunspots and Active Regions}

Local helioseismology has provided intriguing views of the structures and flows under sunspots and active regions. However, there is a growing body of evidence that there are surface effects and other inadequacies of the modelling of wave propagation in these regions that may introduce systematic errors into the present local helioseismic inferences.

The forward problem as applied to the analysis of helioseismic data is currently almost universally based on solving nonmagnetic hydrodynamical equations. Sunspots and active regions are then considered as local perturbations. Most of the analysis to date considers that the sunspot or active region introduces only an isotropic perturbation to the propagation speed of the waves.

Cally and collaborators (see Cally, 2007, and references therein) have emphasised the need for more sophisticated modelling and interpretation of wave propagation in strongly magnetised regions. Their analytical and numerical work highlights several effects on seismic waves, including wave transmission and conversion at the layer where the values of the Alfvén speed and sound speed are equal, and a directional filtering of acoustic waves entering the overlying atmosphere. Work is currently being undertaken by several groups to include MHD effects in numerical simulations of wave propagation suitable for testing local helioseismic techniques.

Using helioseismic holography, Schunker, Braun, and Cally (2007) have found that the measured phase shifts and equivalent travel-time perturbations in sunspots depend on the line-of-sight angle in the plane containing the magnetic field and the local vertical. This appears to be a surface effect. The existence of the effect in time-distance Dopplergram data has been confirmed by Zhao and Kosovichev (2006), who also find however that the effect does not exist for continuum-intensity data. 
Couvidat and Rajaguru (2007) have investigated travel times and associated sound-speed perturbations associated with sunspots. They detect rings of negative mean travel-time perturbations in the travel-time maps of most of the sunspots that they have studied, for travel distances between 8.7 and 11.6 Mm. These rings (see also the top panels in Figure 1) produce significant arclike or ringlike structures in the inversion results, mimicking regions of increased sound speed. The apparent structures are located beneath the sunspot penumbrae and are sensitive to the frequency filtering applied. Couvidat and Rajaguru conclude that the rings are most probably artifacts caused, in a way not yet accounted for, by near-surface interaction between the waves and the magnetic field.

The strong reduction of $p$-mode power in regions of strong surface magnetic field is well known (e.g., Hindman and Brown, 1998; Nicholas, Thompson, and Rajaguru, 2004; and references therein). Possible causes of this reduction include mode conversion and suppression of wave sources within the active region. Rajaguru et al. (2006) have shown that the reduction in acoustic power interacts with a phase-speed filter, if one is applied in the data reduction, to introduce spurious travel-time shifts. An ad hoc solution to reduce this systematic error is to artificially restore the level of the acoustic power signal in the magnetised region before proceeding with the rest of the data analysis. In helioseismic holography, Lindsey and Braun (2003) have coined the term "showerglass effect" for the modulation of amplitudes and phases in regions of strong surface magnetic field.

Jensen, Pijpers, and Thompson (2006) have reported strong asymmetries between crosscorrelation functions corresponding to waves propagating towards or away from regions of strong magnetic field.

Whilst the Gizon - Birch and Gabor wavelet-fitting methods for travel times give results that are in good agreement for quiet-Sun measurements (Roth, Gizon, and Beck, 2007), there may be significant differences when measurements are made in and around sunspots. The wave-packet central frequency obtained from Gabor wavelet fitting is seen to be lower in a sunspot than in the quiet Sun (Figure 2). According to Equation (10), the Gizon - Birch travel times can be sensitive to a shift in central frequency, and hence this may introduce a significant bias compared to measured Gabor-wavelet phase travel times. Evidently, it is important for any inversion in active regions that the kernels used are computed in a manner that is consistent with the definition of the measured travel times. Also Nigam, Kosovichev, and Scherrer (2007) have shown that although the phase-speed filtering procedure does not change the functional form of the basic time - distance Gabor fitting formula (5), it systematically shifts the travel times, if the central phase speed of the filter is different from the actual phase or group speeds for a given distance. Thus, if a change in wave-packet phase speed occurs in a sunspot region, perhaps because of a shift in the frequency of the wave packet (as seen in Figure 2), this might introduce systematic artifacts in the travel times measured using Gabor-wavelet fitting.

One might expect, at least to a first approximation, that any effect of subsurface anomalies on measured travel times would depend on the phase speed of the waves but not on their frequency at fixed phase speed. Braun and Birch (2006) have used helioseismic holography to measure travel-time perturbations within active regions after filtering both according to phase speed and frequency. At fixed phase speed, they find that the inferred travel-time perturbations do depend on frequency. In fact, the functional dependence of the travel-time perturbations on the modes in the region left by the filtering is roughly inversely proportional to mode mass. This is strongly indicative that a significant part of the observed negative travel-time perturbation originates near the surface. In global-mode inversions and some ring-analysis inversions for structure, such a component of the data is explicitly accounted for (Dziembowski, Pamyatnykh, and Sienkiewicz, 1990; Simmons and Basu, 2003). This 

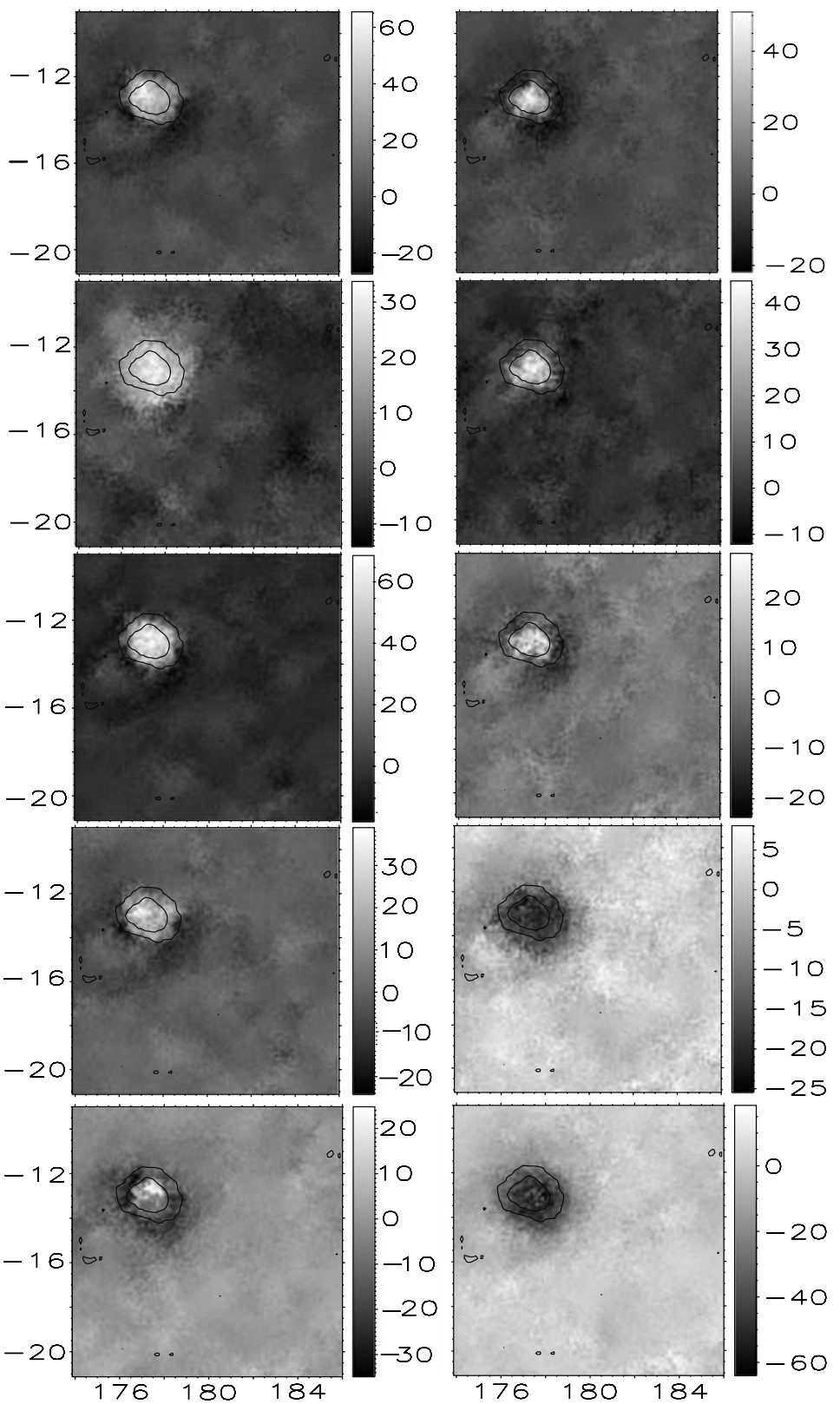

Figure 1 Mean travel times obtained from NOAA 9056 data via Gabor wavelet fitting for skip distances of $7.29 \mathrm{Mm}$ (left column) and $11.664 \mathrm{Mm}$ (right column). The grey scale is in seconds, Carrington longitude is plotted along the $x$-axis, and latitude is along the $y$-axis. The phase-speed filter values were as in Couvidat, Birch, and Kosovichev (2006). Top row: Travel times obtained without frequency bandpass filter. From the second row to the bottom row are the mean travel times obtained by applying additional frequency filters centred at 3.0, 3.5, 4.0, and $4.5 \mathrm{mHz}$. The contours are deduced from the magnetograms and correspond to -150 and -700 gauss. 

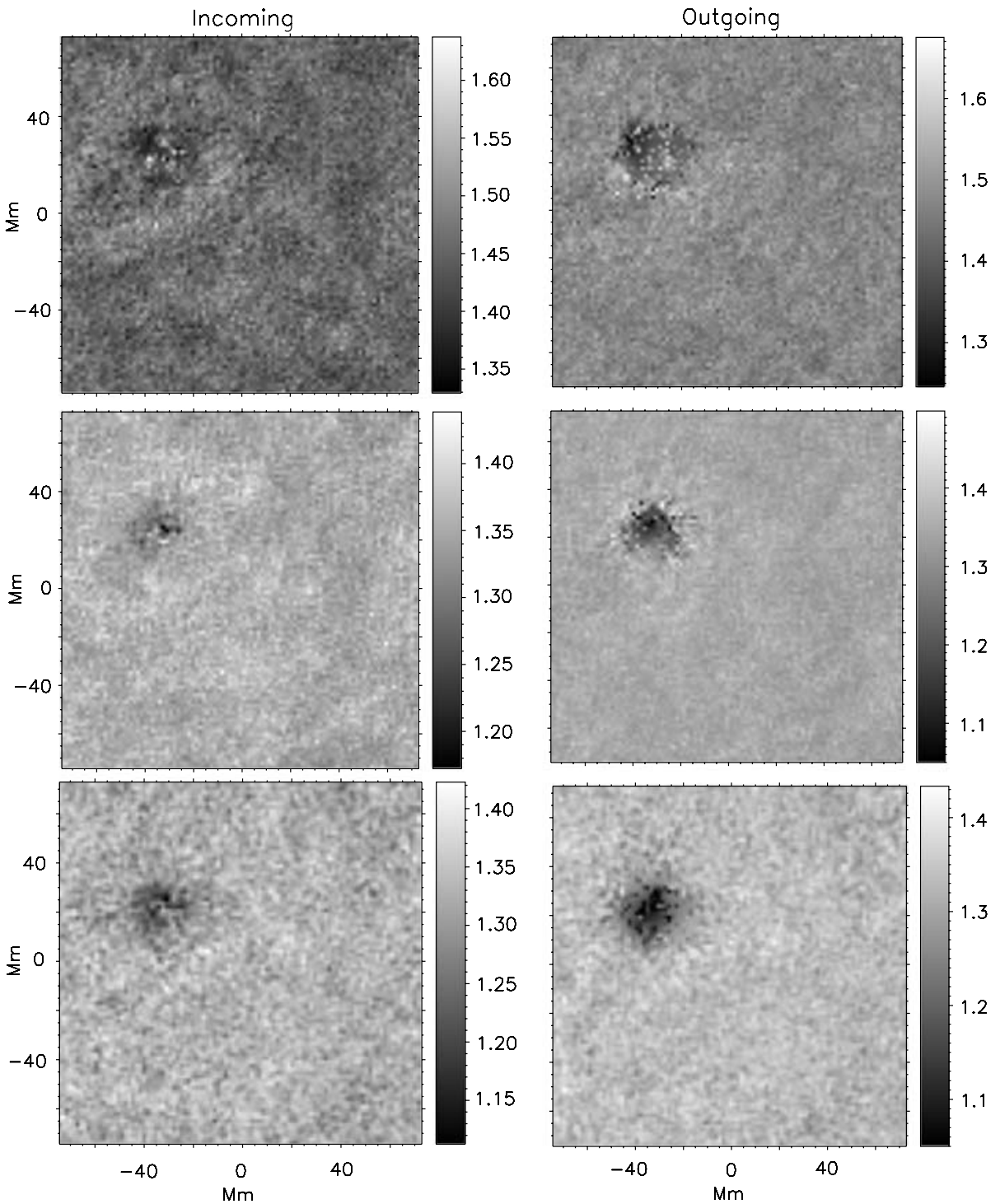

Figure 2 Results of Gabor-wavelet fitting for the central frequency $\left(\omega_{0}\right)$ for incoming (left) and outgoing (right) waves extracted from NOAA $9056 \mathrm{SOHO} / \mathrm{MDI}$ data. The skip distances used are (top to bottom rows) $7.29,11.664$, and 16.038 Mm. The grey scale in all images is in $\min ^{-1}$.

suggests a similar inversion strategy for travel-time perturbations, such that these might be fitted as a sum of a contribution from the interior plus a function $F(v)$ of frequency multiplied by a horizontal weighting function $\Phi$ and inversely weighted by mode mass $\mathcal{M}$ :

$$
\tau=\sum_{\alpha} \int K_{\alpha} \delta q_{\alpha} \mathrm{d} V+\Phi \mathcal{M}^{-1} F(v)
$$




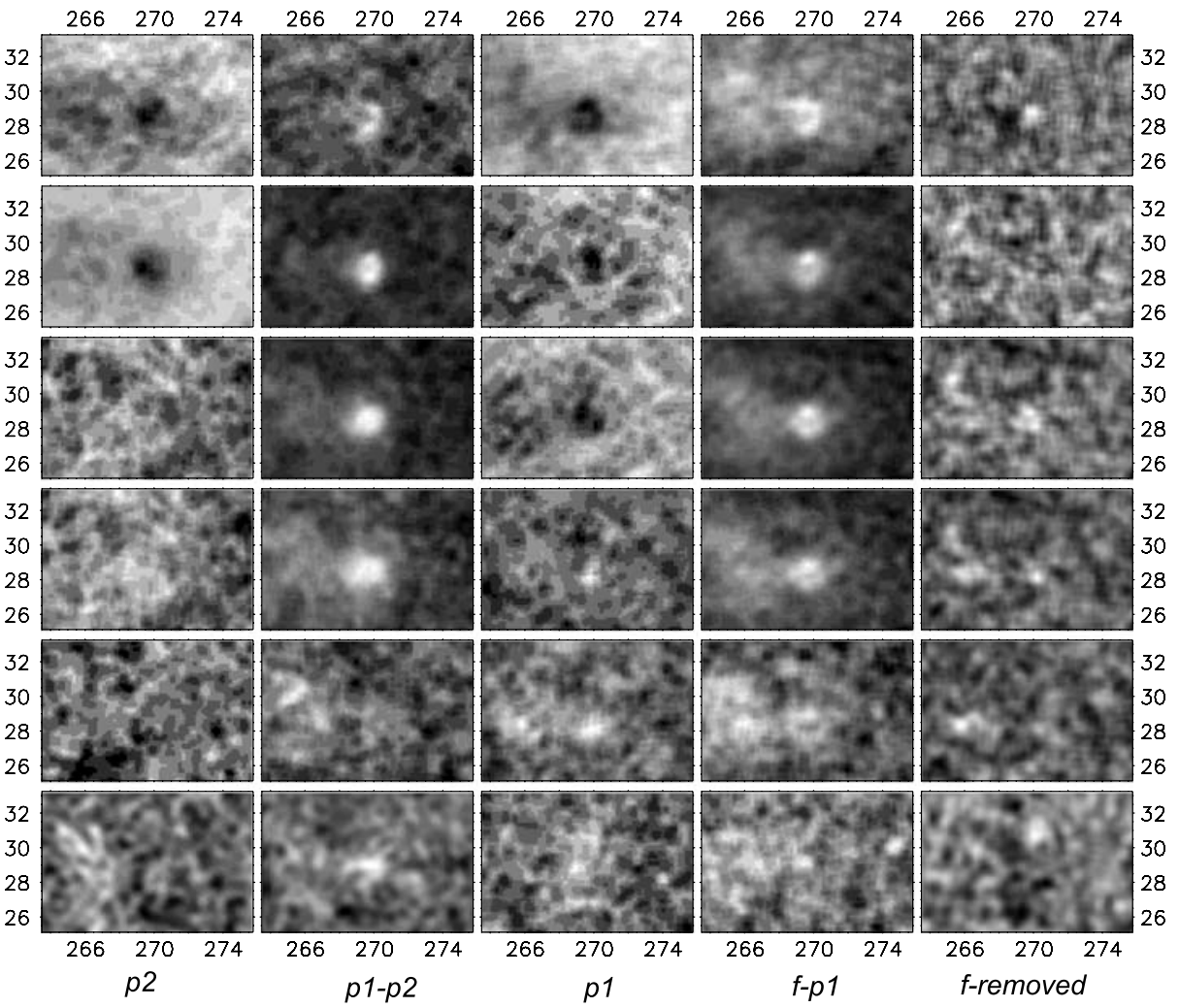

Figure 3 Gizon - Birch travel-time perturbations for isolated sunspot NOAA 9779 obtained for various filtering schemes: Dark tones represent negative perturbations; light tones represent positive perturbations. The skip distance is equal to $5.89 \mathrm{Mm}$. From the right, the columns, respectively, have filters applied as follows: filter to remove the $f$ mode and lower frequencies; pass-filter centred between the $f$ and $p_{1}$ ridges; pass-filter centred on the $p_{1}$ ridge; pass-filter centred between the $p_{1}$ and $p_{2}$ ridges; pass-filter centred on the $p_{2}$ ridge. From the bottom, the rows have bandpass filters centred on $2.0,2.5,3.0,3.5,4.0$, and $4.5 \mathrm{mHz}$, respectively. More details are given in the text.

A further very interesting result has recently been presented by Braun and Birch (2008). This relates to the positive travel-time perturbations obtained for short skip distances in sunspots and widely interpreted as indicating a region of slower wave propagation in the shallow subsurface layers beneath the spot. By investigating where in the space of frequency and phase speed the positive travel-time perturbations show up, and also by applying ridge filters rather than the more usual phase-speed filtering, Braun and Birch find evidence that the positive perturbations arise from the $p_{1}$ ridge or beneath it and are not seen in the higher order $p$-mode data. A similar finding is illustrated in Figures 3 and 4 . Here we present traveltime perturbations measured in the vicinity of isolated sunspot NOAA 9779 relative to the surrounding quiet Sun, using a centre-to-annulus geometry and skip distances of $5.83 \mathrm{Mm}$ (Figure 3) and 16.04 Mm (Figure 4). For each row, a bandpass filter was used to select data within a $1-\mathrm{mHz}$ frequency band with a $0.2-\mathrm{mHz}$ Gaussian roll-off, centred (from bottom to top) at 2.0, 2.5, 3.0, 3.5, 4.0, and $4.5 \mathrm{mHz}$. These were combined for each column (left to right) with filters selecting the data from the $p_{2}$ ridge, in between the $p_{1}$ and $p_{2}$ ridges, the $p_{1}$ ridge, in between $f$ and $p_{1}$ ridges, and finally removing only the $f$-ridge 


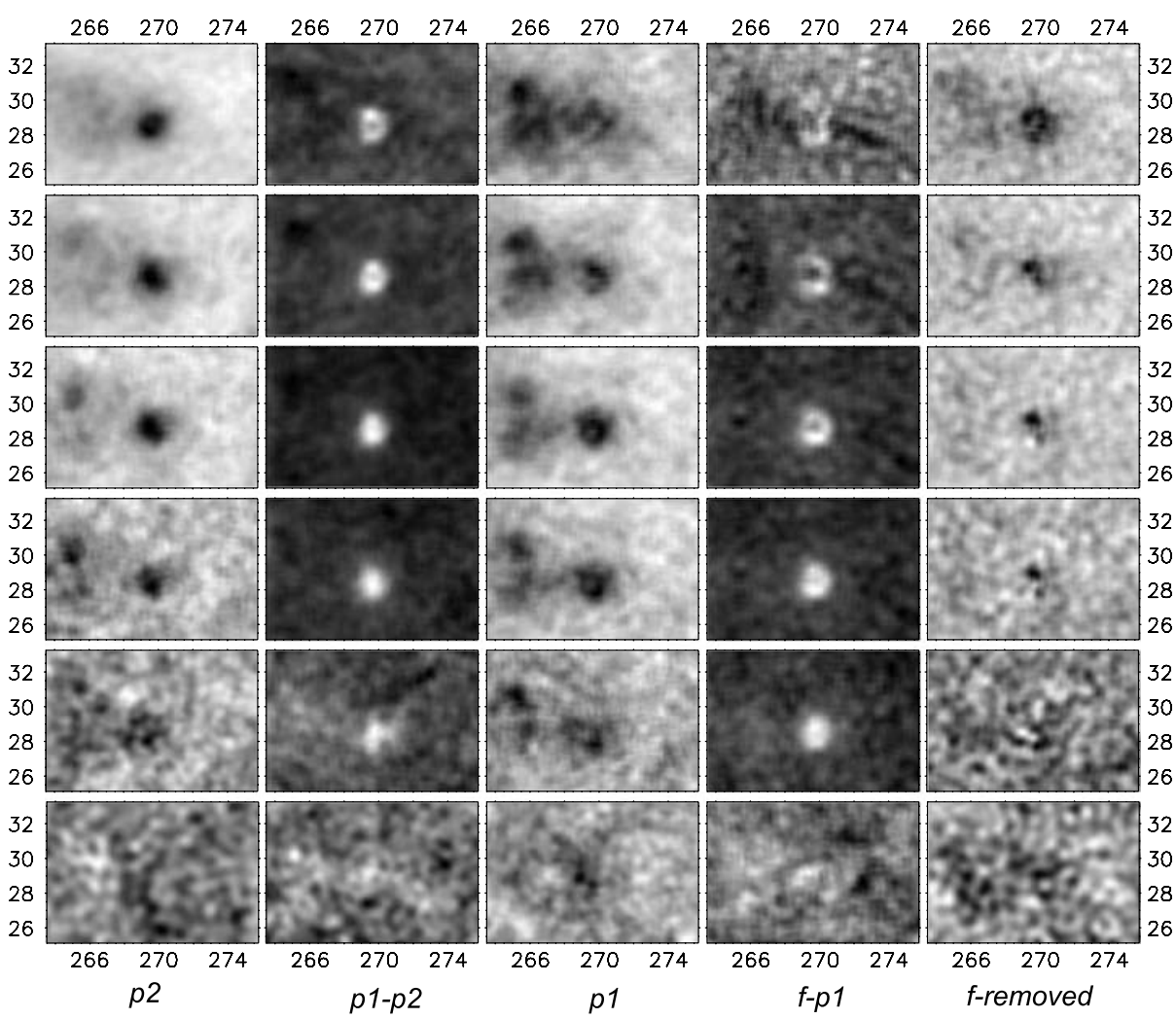

Figure 4 Same as Figure 3, but for a skip distance of 16.038 Mm.

data. The filters were constructed as follows: At constant frequency we apply a filter that takes the value of unity at the horizontal wavenumber corresponding to either a particular ridge, for example, $p_{1}$ (a "ridge filter"), or a midpoint between the adjacent ridges, for example, $p_{1}-p_{2}$ (an "off-ridge filter"). On either side of this centre line the filter has a Gaussian roll-off, with half width at half maximum (HWHM) equal to 0.3175 times the distance to the neighbouring ridge on that side for the ridge filter, and with HWHM equal to 0.625 times the distance to the adjacent ridge in the case of the off-ridge filter. The " $f$-removed" column corresponds to applying simply a standard filter to remove $f$-ridge data as described by Giles (1999). No phase-speed filter was applied. To illustrate the ridge and off-ridge filters, Figure 5 shows the filtered power spectrum for the data, prior to application of the bandpass filters. In agreement with Braun and Birch (2008), as illustrated in Figures 3 and 4, we find a positive travel-time perturbation in the region beneath the $p_{1}$ ridge, but we also find such a signal between the $p_{1}$ and $p_{2}$ ridges and find that on the $p_{1}$ ridge the positive perturbation is absent. Our tentative conclusion is that the positive travel-time perturbation signal arises only in the regions between the $p$-mode ridges and that the travel-time perturbations associated with the data on the ridges themselves are all consistently negative. We do not at present have an explanation for the off-ridge behaviour. 

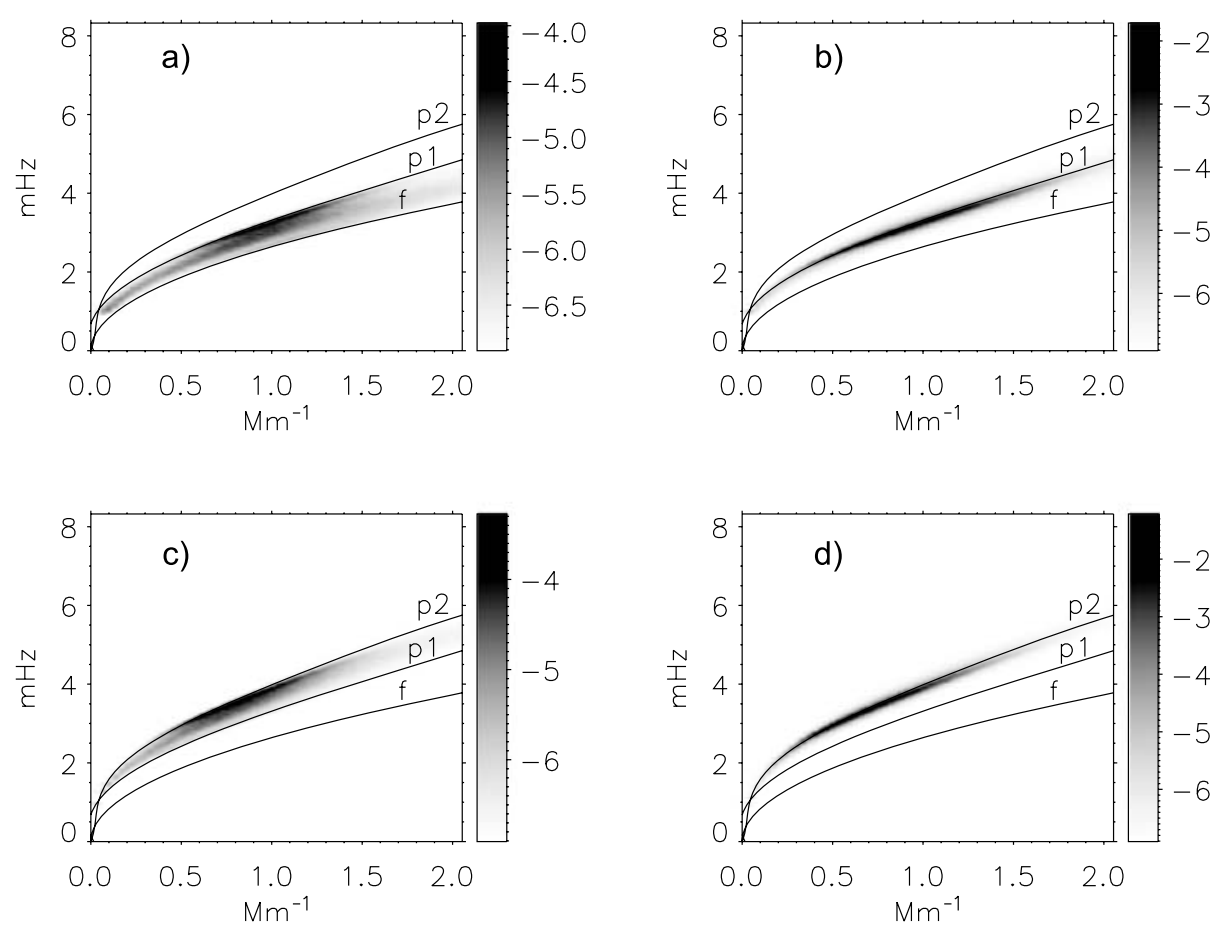

Figure 5 Examples of ridge and off-ridge filters showing the power spectrum after application of, respectively, (a) the $f-p_{1}$ off-ridge filter, (b) the $p_{1}$-ridge filter, (c) the $p_{1}-p_{2}$ off-ridge filter, and (d) the $p_{2}$-ridge filter. The locations of the $f, p_{1}$, and $p_{2}$ ridges, for modes of a theoretical solar model, are indicated by the solid curves. The grey scale is logarithmic with power in arbitrary units.

\section{Simulations}

Recent progress in realistic simulation of the solar interior, in particular of the upper convection zone, is providing opportunities to test the accuracy and robustness of local helioseismic techniques.

Zhao et al. (2007) have used the three-dimensional, radiative-hydrodynamic simulations of the upper convection zone and photosphere of Benson, Stein, and Nordlund (2006) to evaluate the results of time-distance inversion in quiet-Sun regions. The waves are generated consistently within the simulations. The horizontal-flow fields inferred from inverting $f$-mode travel times are in good agreement with the actual flows in the simulations, down to $3 \mathrm{Mm}$ depth.

Other work has introduced flows into numerical simulations of wave propagation to see how reliably the flows can be recovered. Shelyag, Erdélyi, and Thompson (2007) tested ray-theory application to the flows in the quiet Sun. They find that most of the error in a ray-theoretic inversion result can be understood to arise from the sensitivity of the waves to the flows over a greater depth than is accounted for by the ray paths.

Braun et al. (2007) have applied helioseismic holography to the simulations by Benson, Stein, and Nordlund (2006). They obtain phase shifts, which they then converted to traveltime perturbations. The holography recovers the model travel times well, consistent with the noise in the simulated measurements. Moreover, the travel times have been measured in different frequency bands and compared with the model predictions from kernels computed 
in the Born approximation: The level of agreement gives a fair degree of confidence in the kernels for the kinds of flows in the study. Also in the context of helioseismic holography, Birch, Braun, and Hanasoge (2008) have conducted simulations to investigate the sensitivity of measured travel times to choice of filter parameters (as discussed for observational data in Section 4).

In the previous section it was remarked that the acoustic power is observed to be reduced in regions of strong magnetic field. This reduction might be caused by a lack of granulationrelated wave sources in the region. This has been demonstrated in numerical simulation of wave propagation, in which reduction of the amplitudes of random wave sources in a circular region corresponding to a sunspot gives rise to a reduction in the oscillation amplitude in the region by a factor of two to four (Parchevsky and Kosovichev, 2007). Hanasoge et al. (2008) have explored the effects on travel times: By suppressing the sources, these authors have shown in their simulations that meaningful travel times depend strongly on the homogeneity of sources in the medium, with asymmetric ingoing and outgoing wave travel-time perturbations being obtained. The results of Hanasoge et al. appear to be in very good agreement with the cross-correlation asymmetries observed around an active region by Jensen, Pijpers, and Thompson (2006).

It is essential for testing inferences in strongly magnetic regions that MHD simulations are developed. Such a simulation code has now been developed for studying the interaction of linear waves with magnetic structures in an inhomogeneous atmosphere (Cameron, Gizon, and Daiffallah, 2007) and has already been used, for example, to compare observed cross-correlations on the Sun with the interaction of a simulated plane wave with a magnetic flux cylinder.

\section{Prospects}

Local helioseismology has enormous potential and has already produced many intriguing insights into the subsurface layers of the Sun. We have touched on several areas of application. Although we have not discussed it here, there have also been a number of recent papers resulting from the holographic study of the seismic response of the solar interior to flares, which should improve our understanding of flare physics (Donea, 2006; Kosovichev, 2006; Kosovichev, 2007; Martinez-Oliveros et al., 2007; Moradi et al., 2007; Zharkova and Zharkov, 2007).

In regions of the quiet Sun, comparative studies using more than one method and studies using numerical simulations are leading to increasing confidence in the reliability of the helioseismic findings.

In regions of strong surface magnetic field, however, there is evidence from observation and from theory that our forward modelling and data analysis techniques are not yet developed well enough to allow robust conclusions about the subsurface structure, nor possibly about the flows. Nonetheless, we are optimistic that we shall see substantial advances in the next year or two. The analytical and simulations work is progressing rapidly and needs now to inform the forward modelling used in interpreting the data. The observations are giving a strong lead to the theorists as to the areas where improved understanding is needed and may also suggest data analysis strategies that are less sensitive to uncertain aspects of the modelling.

Soon a wealth of high-resolution data for local helioseismology will be available from the HMI instrument onboard the Solar Dynamics Observatory, covering the entire solar disk. The SOT instrument onboard Hinode, with high resolution and a pointing capability, 
will enable seismic studies (Mitra-Kraev, Kosovichev, and Sekii, 2008) of small regions including at high latitudes. No doubt these will provide new challenges as well as insights both for our techniques and for our understanding of the Sun.

Acknowledgements We thank Aaron Birch, Doug Braun, Laurent Gizon, and the referee for helpful comments that have improved this paper. This work was supported by the UK Science and Technology Facilities Council through Grant Nos. PP/C502914/1 and PP/B501512/1 and by the European Helio- and Asteroseismology Network (HELAS), a major international collaboration funded by the European Commission's Sixth Framework Programme.

\section{References}

Basu, S., Antia, H.M., Tripathy, S.C.: 1999, Astrophys. J. 512, 458.

Benson, D., Stein, R., Nordlund, A: 2006, In: Uitenbroek, H., Leibacher, J., Stein, R.F. (eds.) Solar MHD Theory and Observations: A High Spatial Resolution Perspective CS-354, Astron. Soc. Pac., San Francisco, 92.

Birch, A.C.: 2006, In: Fletcher, K., Thompson, M. (eds.) Beyond The Spherical Sun, Proc SOHO 18/GONG 2006/HELAS I SP-624, ESA, Noordwijk, 2.1, publ on CDROM.

Birch, A.C., Kosovichev, A.G.: 2000, Solar Phys. 192, 193.

Birch, A.C., Kosovichev, A.G., Duvall, T.L. Jr.: 2004, Astrophys. J. 608, 580.

Birch, A.C., Braun, D.C., Hanasoge, S.M.: 2008, Solar Phys. in press.

Birch, A.C., Gizon, L., Hindman, B.W., Haber, D.A.: 2007, Astrophys. J. 662, 730.

Braun, D.C., Birch, A.C.: 2006, Astrophys. J. 647, 187.

Braun, D.C., Birch, A.C.: 2008, Solar Phys. accepted.

Braun, D.C., Duvall, T.L. Jr., Labonte, B.J.: 1987, Astrophys. J. 319, L27.

Braun, D.C., Birch, A.C., Benson, D., Stein, R.F., Nordlund, Å.: 2007, Astrophys. J. 669, 1395.

Cally, P.S.: 2007, Astron. Nachr. 328, 286.

Cameron, R., Gizon, L., Daiffallah, K.: 2007, Astron. Nachr. 328, 313.

Chou, D.-Y.: 2000, Solar Phys. 192, 241.

Couvidat, S., Rajaguru, S.P.: 2007, Astrophys. J. 661, 558.

Couvidat, S., Birch, A.C., Kosovichev, A.G.: 2006, Astrophys. J. 640, 516.

Donea, A.: 2006, Solar Phys. 239, 113.

Duvall, T.L. Jr., Birch, A.C., Gizon, L.: 2006, Astrophys. J. 646, 553.

Duvall, T.L. Jr., Jefferies, S.M., Harvey, J.W., Pomerantz, M.A.: 1993, Nature 362, 430.

Duvall, T.L. Jr., Scherrer, P.H., Bogart, R.S., Bush, R.I., De Forest, C., Hoeksema, J.T., Schou, J., Saba, J.L.R., Tarbell, T.D., Title, A.M., Wolfson, C.J., Milford, P.N.: 1997, Solar Phys. 170, 63.

Dziembowski, W.A., Pamyatnykh, A.A., Sienkiewicz, R.: 1990, Mon. Not. Roy. Astron. Soc. 244, 542.

Giles, P.M.: 1999, Ph.D. dissertation, Stanford University.

Giles, P.M., Duvall, T.L. Jr., Scherrer, P.H., Bogart, R.S.: 1997, Nature 390, 52.

Gizon, L., Birch, A.C.: 2002, Astrophys. J. 571, 966.

Gizon, L., Birch, A.C.: 2004, Astrophys. J. 614, 472.

Gizon, L., Birch, A.C.: 2005, Living Rev. Solar Phys. 2. http://solarphysics.livingreviews.org/Articles/ Irsp-2005-6/.

Gizon, L., Thompson, M.J.: 2007, Astron. Nachr. 328, 204.

Gizon, L., Duvall, T.L. Jr., Larsen, R.M.: 2001, In: Brekke, P., Fleck, B., Gurman, J. (eds.) Proc. $23 r d$ IAU General Assembly, 203, Astron. Soc. Pac., San Francisco, 189.

Haber, D.A., Hindman, B.W., Toomre, J., Bogart, R.S., Hill, F.: 2001, In: Wilson, A. (ed.) Helio- and Asteroseismology at the Dawn of the Millennium, Proc. SOHO 10/GONG 2000 SP-464, ESA, Noordwijk, 209.

Hanasoge, S.M., Couvidat, S., Rajaguru, S.P., Birch, A.C.: 2008, Astrophys. J. submitted. arXiv:astro$\mathrm{ph} / 0707.1369 \mathrm{v} 3$.

Hill, F.: 1988, Astrophys. J. 333, 996.

Hindman, B.W., Brown, T.M.: 1998, Astrophys. J. 504, 1029.

Hindman, B.W., Gough, D., Thompson, M.J., Toomre, J.: 2005, Astrophys. J. 621, 512.

Jackiewicz, J., Gizon, L., Birch, A.C., Duvall, T.L. Jr.: 2007, Astrophys. J. 671, $1051-1064$.

Jensen, J.M., Pijpers, F.P.: 2003, Astron. Astrophys. 412, 257.

Jensen, J.M., Pijpers, F.P., Thompson, M.J.: 2006, Astrophys. J. 648, 75.

Kosovichev, A.G.: 2006, Solar Phys. 238, 1. 
Kosovichev, A.G.: 2007, Astrophys. J. 670, L65.

Kosovichev, A.G., Duvall, T.L.: 1997, In: Pijpers, F.P., Christensen-Dalsgaard, J., Rosenthal, C.S. (eds.) SCORe'96: Solar Convection and Oscillations and Their Relationship, ASSL 225, Kluwer Academic, Dordrecht, 241.

Kosovichev, A.G., Duvall, T.L. Jr., Scherrer, P.H.: 2000, Solar Phys. 192, 159.

Lindsey, C., Braun, D.C.: 1997, Astrophys. J. 485, 895.

Lindsey, C., Braun, D.C.: 2000, Science 287, 1799.

Lindsey, C., Braun, D.C.: 2003, In: Sawaya-Lacoste, H. (ed.) Local and Global Helioseismology: The Present and Future, Proc SOHO 12/GONG+ 2002 SP-517, ESA, Noordwijk, 23.

Martinez-Oliveros, J.C., Moradi, H., Besliu-Ionescu, D., Donea, A.-C., Cally, P.S., Lindsey, C.: 2007, Solar Phys. 245, 121.

Mitra-Kraev, U., Kosovichev, A.G., Sekii, T.: 2008, Astron. Astrophys. Lett. accepted. arXiv:astro$\mathrm{ph} / 0711.2210 \mathrm{v} 1$.

Moradi, H., Donea, A.-C., Lindsey, C., Besliu-Ionescu, D., Cally, P.S.: 2007, Mon. Not. Roy. Astron. Soc. 374, 1155 .

Nicholas, C.J., Thompson, M.J., Rajaguru, S.P.: 2004, Solar Phys. 225, 213.

Nigam, R., Kosovichev, A.G., Scherrer, P.H.: 2007, Astrophys. J. 659, 1736.

Parchevsky, K., Kosovichev, A.G.: 2007, Astrophys. J. 666, L53.

Rajaguru, S.P., Birch, A.C., Duvall, T.L. Jr., Thompson, M.J., Zhao, J.: 2006, Astrophys. J. 646, 543.

Roth, M., Gizon, L., Beck, J.G.: 2007, Astron. Nachr. 328, 215.

Schunker, H., Braun, D.C., Cally, P.S.: 2007, Astron. Nachr. 328, 292.

Shelyag, S., Erdélyi, R., Thompson, M.J.: 2007, Astron. Astrophys. 469, 1101.

Simmons, B., Basu, S.: 2003, In: Sawaya-Lacoste, H. (ed.) Local and Global Helioseismology: The Present and Future, Proc. SOHO 12/GONG+ 2002 SP-517, ESA, Noordwijk, 393.

Woodard, M.F.: 2002, Astrophys. J. 565, 634.

Zhao, J., Kosovichev, A.G.: 2004, Astrophys. J. 603, 776.

Zhao, J., Kosovichev, A.G.: 2006, Astrophys. J. 643, 1317.

Zhao, J., Georgobiani, D., Kosovichev, A.G., Benson, D., Stein, R.F., Nordlund, Å.: 2007, Astrophys. J. 659, 848.

Zharkova, V.V., Zharkov, S.I.: 2007, Astrophys. J. 664, 573. 